\title{
Conceptual design decision support of a mechatronic system using analytical approach with Modelica
}

\author{
Ghazoi Hamza ${ }^{1,2,}$, Moncef Hammadi ${ }^{1}$, Maher Barkallah ${ }^{2}$, Jean-Yves Choley ${ }^{1}$, Alain Riviere ${ }^{1}$, Jamel Louati ${ }^{2}$, and \\ Mohamed Haddar ${ }^{2}$ \\ ${ }^{1}$ QUARTZ EA7393, SUPMECA-Paris, 3 rue Fernand Hainaut, Saint-Ouen 93407, France \\ 2 Mechanics, Modeling and Production Research Laboratory, National School of Engineers of Sfax, University of Sfax, BP.1173, \\ Sfax 3038, Tunisia
}

Received: 16 May 2017 / Accepted: 4 March 2018

\begin{abstract}
During conceptual design phase, system architects pre-validate architectures following different constrains. This paper proposes a new pre-designing method applied to a flexible multibody system to characterize its vibrational behavior analytically with Dymola/Modelica language. We study the vibration interaction between perturbations sources (motors) and receivers (electronic cards) through a flexible beam with arbitrary boundary conditions. In fact, a lot of mechatronic systems may have their natural frequencies and mode shapes dependent on their spatial configuration, which affect the dynamic response of the system. This methodology shows that using Modelica, we can implement different boundary conditions, making the element suitable for any multibody simulation. This model can help system architects to study the influence of the boundary conditions on the system response. Two types of boundary conditions are considered in this study, simply supported-simply supported (S-S) and clamped-clamped (C-C). The solution for this issue would be a pre-positioning procedure which targets to provide a support for decision-making on the feasibility of a given system architecture at an early design stage, before proceeding to the detailed design. The simulation results have been validated with respect to the literature and by a comparison with those obtained by the model based on the Beam component which belongs to the Modelica flexible Bodies Library.
\end{abstract}

Keywords: Pre-designing / Dymola / vibration / flexible multibody system

\section{Introduction}

The operation of most industrial systems involves interactions between several physical phenomena of different natures (mechanical, thermal, electromagnetic, etc.) [1]. These systems, called mechatronic systems, target to reconcile all these design constraints and to combine competing technologies by integrating them in synergy. Their appearance makes essential a change of methods in the design process and verification of the overall system performance [2]. Usually, the first actions taken in engineering are: technical specification of requirement, preconception and research of technological solutions and then detailed design.

The preconception phase (conceptual design phase) is considered as one of the most significant design phase in the process of systems engineering [3-5]. In this stage, designers select concepts according to the user's needs, that will be employed in solving a given design problem and decide how

\footnotetext{
* e-mail: hamza.ghazoi@gmail.com
}

to interconnect these concepts into a suitable system architecture [6]. The selections made in this phase will guide all design activities during the detailed design phases $[7,8]$.

In the first step of architectural choices (or pre-design), a skeleton of the mechanism is defined. The geometric models used are often very simple. Simplification makes it possible to use much simpler and faster analysis methods. In order to reduce the complexity of the models, it is desirable to start with a description of the higher level of the system.

The component position is performed usually at the end of the design process during the detailed design. However preceding considerations show how important is to take into account as soon as possible component positioning and geometry to design mechatronic systems [1,9].

The design of mechatronic systems is often a complex problem, as it is based on the integration of several disciplines, such as mechanical and electrical. Decomposition is therefore a common technique to help designers to obtain solutions for the design of mechatronic systems during the systems engineering process. To study such a 
system, it is then required a way to break it down into interconnected, smaller sub-problems, and then to join them together [3]. The concept of breaking down a problem into smaller pieces is then important to study a mechatronic system.

Currently, an important focus is on the development and implementation of numerical methods to simulate physical phenomena. Unfortunately, the vibratory analysis in the preliminary mechatronic design is rarely considered essential. So many vibration studies are carried out in the detailed design level or even after the manufacture of the systems.

In mechatronic design, the majority of complex structures are formed by structural elements of relatively simple topology such as beams, plates and shafts $[10,11]$. Today, the most widely used approach to model vibrational behavior of these structures is the finite element method. This approach is effective; but, it is very expensive in space memory and in computing time and it also do not allow an analysis of the complete mechatronic system, not only for of the big number of components to be integrated, but also the necessity to integrate functionalities such as control, which is difficult to model with the finite element method.

Considerable attempts have been made recently to implement the vibration behavior of mechatronic systems taking into account the dynamic flexibility of mechanical subsystems. For example, the discrete-continuous vibrating mechatronic system was considered in $[12,13]$. The Galerkin's method is used to study the dynamic characteristics of the system. The flexural vibrating one-dimensional mechatronic system was considered in [14]. In fact, piezoelectric transducer bonded on cantilever beam, the beam is loaded with force, which operates perpendicular to the beam axis. The influence of different parameters of the components on the dynamic characteristics of the system has been studied.

In the previous research works of the authors, a method for the preliminary design of a mechatronic system has been proposed $[15,16]$. In fact, we study the vibration transmission between electromechanical components (motors and electronic cards) which are located on a simply supported rectangular plate. We consider that the motor induces a dynamic excitation over a rectangular finite area. The electronic card is modeled as a rigid mass mounted resiliently over the vibrating plate [17-19]. Several parameters having significant influence on the physical interaction have been analyzed in order to show the aptitude of the model to help designers at an early stage of design. The preliminary dimensioning of mechatronic systems does not require computational models or a large amount of data which are cumbersome to construct and maintain, especially in the case of a mechatronic system. The analytical approach modeling is then very appropriate at this stage as it requires the least computer resources. The main aim of this work is to present a pre-designing method, based on the normal mode approach (analytical method), to help system architects to select concepts and dimensions of the components and to study the physical interactions at an early stage of design. Our contribution is organized as follows. Section 2 provides the analytic formulation of the problem. Section 3 describes the system modeling, Section 4 presents some results and Section 5 summarizes and concludes the paper.

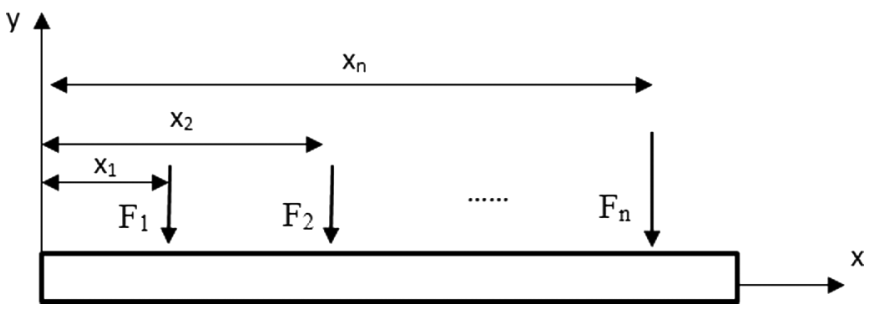

Fig. 1. Structural system subjected to forced vibration.

\section{The dynamic flexibility of the mechanical subsystem}

\subsection{Analytic formulation of the beam vibration}

The considered system (Fig. 1) consists of beam which has a square cross-section. Before developing the analytical model, the following assumptions were made: Bernoulli Euler theory of vibration of thin beams was considered so the effects of rotary inertia and shear deformation are neglected. The beam is also elastic, isotropic with homogeneous constitutive material. The system vibration is harmonic. In this study, only transverse vibration is considered. The normal mode approach (modal analysis) used in reference [20] will be adopted here.

The dynamics of its deflection curve $w(x, t)$ is described by the partial differential equation (PDE) given by [20]:

$$
E I \frac{\partial^{4} w(x, t)}{\partial x^{4}}+\rho A \frac{\partial^{2} w(x, t)}{\partial t^{2}}=f(x, t),
$$

where $E$ denotes the Young's modulus, $\rho$ is the mass density of the beam material, $I$ is the area moment of inertia, $A$ is the area of the beam cross-section, $t$ is time, $f(x, t)$ is the timevarying force per unit length and $l$ is the beam length.

We consider that every motor mounted on the beam induce a dynamic excitation in a well-defined position. In this case, we consider a concentrated force $p_{k} \sin \left(\Omega_{k} t\right)$ type force acts in a point $x_{k}$, equivalent to the excitation generated by the motor. $\Omega_{k}$ is the angular frequency of the dynamic excitation and $F_{k}$ is the dynamic force intensity.

The transverse external loadings are:

$$
f(x, t)=\sum_{k=1}^{N} F_{k} \sin \left(\Omega_{k} t\right) \delta\left(x-x_{k}\right),
$$

with $N$ is the electric motors number.

The solution of the beam's differential equation is assumed to be expressible in the form of this series [20]:

$$
w(w, t)=\sum_{i=1}^{\infty} W_{i}(x) \eta_{i}(t)
$$

where $W_{i}(x)$ are the normal modes, $\eta_{i}(t)$ are the generalized coordinates

Substituting equation (3) into equation (1) resulting in:

$$
\frac{\frac{d^{4} W_{i}(x)}{d x^{4}}}{W_{i}(x)}=\frac{-\rho A}{E I} \frac{\frac{d^{2} \eta_{i}(t)}{d t^{2}}}{\eta_{i}(t)}=\beta_{i}^{4} .
$$


When a dynamic excitation is applied to the beam, the solution of equation (1) takes this form [20]:

$$
\begin{gathered}
w(x, t)=\sum_{i=1}^{\infty}\left[A_{i} \cos \omega_{i} t+B_{i} \sin \omega_{i} t+\frac{1}{\omega_{i}} \int_{0}^{t} Q_{i}(\tau) \sin \omega_{i}\right. \\
(t-\tau) d \tau] W_{i}(x),
\end{gathered}
$$

where the generalize force $Q_{i}(t)$ corresponding to the $i$ th mode takes the form:

$$
Q_{i}(t)=\int_{0}^{l} W_{i}(x) f(x, t) d x, \quad i=1,2, \ldots
$$

The natural frequency of a certain vibration mode can be expressed as follows [20]:

$$
\omega_{i}=\left(\beta_{i} l\right)^{2} \sqrt{\left(\frac{E I}{\rho A l^{4}}\right)},
$$

where the product $\beta_{k} l$ is the $i$ th root of the characteristic frequency equation.

For the resolution of these equations, it is necessary to know the boundary conditions. We propose the study of beams which connections at the ends are different. Two combinations of boundary conditions have been considered in this investigation: clamped at both ends beam $(\mathrm{C}-\mathrm{C})$ and simply supported at both ends beam (S-S).

For a clamped at both ends (C-C) beam, the boundary conditions are:

$$
W(0)=0, \frac{d W}{d x}(0)=0, W(l)=0, \frac{d W}{d x}(l)=0,
$$

The normal modes can be expressed as [20]:

$$
W_{i}(x)=\left[\begin{array}{c}
\left(\cos \beta_{i} x-\cosh \beta_{i} x\right)-\frac{\cos \beta_{i} l-\cosh \beta_{i} l}{\sin \beta_{i} l-\sinh \beta_{i} l} \\
\left.\left(\sin \beta_{i} x-\sinh \beta_{i} x\right)\right] .
\end{array}\right.
$$

And, the eigenvalues $\beta_{i} l$ of the $(\mathrm{C}-\mathrm{C})$ beam can be determined through

$$
\begin{gathered}
\cos \left(\beta_{i} l\right) \cosh \left(\beta_{i} l\right)-1=0, \quad i=1,2,3 \ldots, \\
\text { then } \quad \beta_{i} l=\frac{2 i+1}{2} \pi .
\end{gathered}
$$

For a (S-S) beam, the boundary conditions are:

$$
\begin{aligned}
W(0) & =0, E I \frac{d^{2} W}{d x^{2}}(0)=0, w(l)=0, E I \frac{d^{2} W}{d x^{2}}(l) \\
& =0 .
\end{aligned}
$$

The normal modes take the form [20]:

$$
W_{i}(x)=\sqrt{\frac{2}{\rho A l}} \sin \frac{i \pi x}{l} .
$$

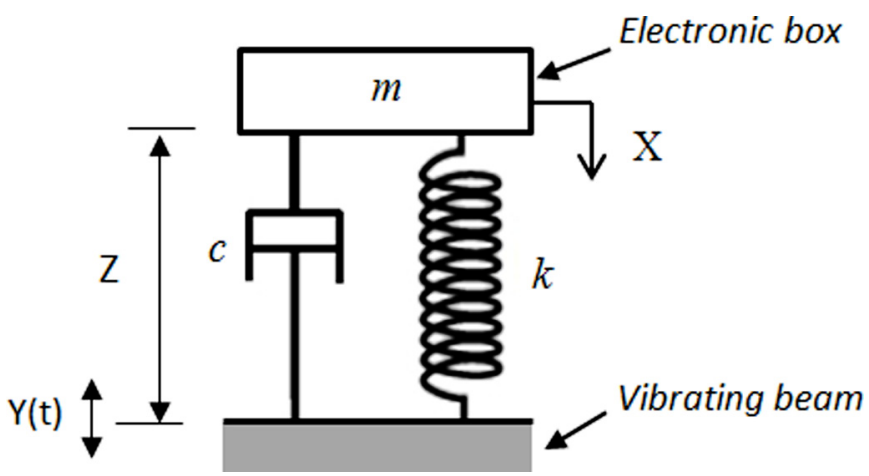

Fig. 2. Equivalent model of the vibrating electronic card.

The eigenvalues $\beta_{i} l$ of a $(\mathrm{S}-\mathrm{S})$ beam can be determined through

$$
\sin \left(\beta_{i} l\right)=0 \quad \text { then } \quad \beta_{i} l=i \pi .
$$

For zero initial condition, the generalized coordinates in the ith mode become [20]:

$$
\begin{aligned}
\eta_{i}(t) & =\frac{1}{\omega_{i}} \int_{0}^{t} Q_{i}(\tau) \sin \omega_{i}(t-\tau) d \tau \\
& =\frac{p_{k}}{\omega_{i}} \int_{0}^{t} W_{i}\left(x_{k}\right) \sin \Omega_{k} \tau \sin \omega_{i}(t-\tau) d \tau \\
& =\frac{F_{k}}{\omega_{i}^{2}} W_{i}\left(x_{k}\right) \frac{1}{1-\Omega^{2} /{ }_{\omega}{ }^{2}}\left(\sin \Omega_{k} t-\frac{\Omega_{k}}{\omega_{i}} \sin \omega_{i} t\right) .
\end{aligned}
$$

Based on the superposition method, the response of the beam can be expressed as:

$$
w(x, t)=\sum_{k=1}^{N} F_{k} \sum_{i=1}^{\infty} \frac{W_{i}(x) W_{i}\left(x_{k}\right)}{\omega_{i}^{2}-\Omega_{k}^{2}}\left(\sin \Omega_{k} t-\frac{\Omega_{k}}{\omega_{i}} \sin \omega_{i} t\right) .
$$

\subsection{Electronic card modeling}

The electronic card can be modelled as the simple dynamically equivalent model shown in Figure 2. It is a rigid mass supported by a linear spring and damper $[17,18,21]$. The electronic card is mounted resiliently over the beam. The movement of the base is $Y(t)$.

The equation of relative motion of the system is expressed in the form:

$$
Z+2 \sigma \xi \dot{Z}+\sigma^{2} Z=-Y
$$

$Z$ is the relative displacement of the electronic card with respect to the beam.

where $\dot{Z}$ is the first derivative of the displacement with respect to time (velocity) and $Z$ is the second derivative of the displacement with respect to time.

$$
Z=X-Y \text {. }
$$

$\sigma=\sqrt{\frac{k}{m}}$ is the angular frequency, $\mathrm{m}$ is the moving mass and $k$ is the spring stiffness. 
The loss factor $\xi=\frac{c}{C_{c}}$ with, $\mathrm{c}$ is the damping coefficient and $C_{c}=2 m \sigma$.

The dynamic response of the beam is expressed as follows:

$$
\begin{aligned}
Y(x, t) & =w(x, t) \\
& =\sum_{k=1}^{N} F_{k} \sum_{i=1}^{\infty} \frac{W_{i}(x) W_{i}\left(x_{k}\right)}{\omega_{i}^{2}-\Omega_{k}^{2}}\left(\sin \Omega_{k} t-\frac{\Omega_{k}}{\omega_{i}} \sin \omega_{i} t\right) .
\end{aligned}
$$

For two motors, the beam displacement can be written as:

$$
\begin{aligned}
Y(x, t) & =w(x, t) \\
& =F_{1} \sum_{i=1}^{\infty} \frac{W_{i}(x) W_{i}\left(x_{1}\right)}{\omega_{i}^{2}-\Omega_{1}{ }^{2}}\left(\sin \Omega_{1} t-\frac{\Omega_{1}}{\omega_{i}} \sin \omega_{i} t\right) \\
& +F_{2} \sum_{i=1}^{\infty} \frac{W_{i}(x) W_{i}\left(x_{2}\right)}{\omega_{i}^{2}-\Omega_{2}{ }^{2}}\left(\sin \Omega_{2} t-\frac{\Omega_{2}}{\omega_{i}} \sin \omega_{i} t\right) .
\end{aligned}
$$

\section{Modelica system modeling}

In this paper, the object-oriented simulation software Dymola/Modelica has been used to develop and simulate this system (Fig. 3). According to the theoretical analysis presented in Section 2, two motors and an electronic card located on the beam are considered in this study.

In order to study the physical interaction and to define the components location, a mechatronic system must be divided into its components. As Modelica is an object oriented modeling language, it allows the realization of a complex system by simple gathering components [22].

The proposed model has been implemented in Dymola/ Modelica. Figure 4 is a graphic representation of the model designed in Dymola. From a structural view point, the setup of the system is decomposed into a set of connected components. It includes elements developed specifically for this application and parts that belong to standard Modelica library.

The beam is modeled by the behaviour characteristic equations presented in Section 2. Two selected boundary conditions are considered in this study: clamped at both ends (C-C) beam and simply supported at both ends (S-S) beam. The user has the ability to choose particular boundary conditions for simulating the flexural deformation and he can also compare the behavior response of a system for the two types of boundary conditions in the same time. Beam parameters are set in graphic interface to define measures and properties of the object. To attach the beam to other system components, the standard Modelica flange connector from the translational library is used. In fact, the beam is equipped with two flange type connectors which are Flange a and Flange $b$ in order to describe the physical interface between respectively, the $(\mathrm{S}-\mathrm{S})$ beam and the (C-C) beam with other components.

The motor has been modeled as a dynamic excitation over the beam and its position on the beam is parameterized.

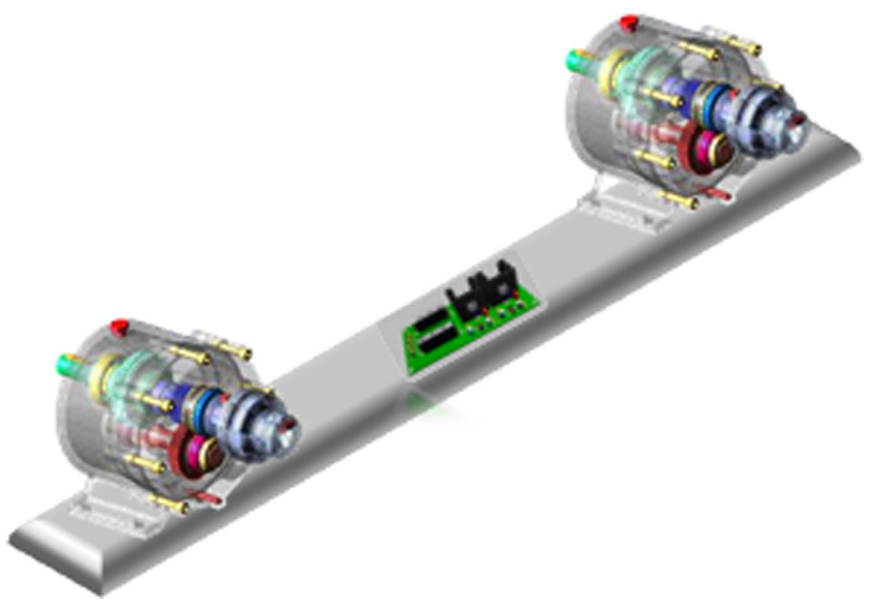

Fig. 3. The studied model.

Models coupling are realized due to the connectors. In fact, each component is equipped with one or more connectors (often also called "ports") which may have different natures in order to describe the interaction possibilities. All quantities needed to describe the interaction circulate in a well-defined and known sense. We have developed a new connector in Modelica/Dymola in order to describe the physical interaction between the beam and the Motor. The characteristics of the new connector are presented in Table 1 . The connector contains three variables which are the angular velocity, the Force magnitude and the motor position over the beam.

Components of the Modelica library are used: 1D model composed of a spring and damper in parallel and the sliding mass, to model the electronic card. Sensors, belonging to the Modelica standard library, are used to control the displacement, the speed, and the acceleration of the electronic card on the beam. The physical system is then constructed by connecting the components through their connectors. The important feature of this system modeling is the component-oriented modeling is based on the solution of mixed continuous-discrete systems of equations. In fact, the continuous model is represented by using PDEs however the discrete model is based on the ordinary differential of equations. By giving parameter values in models dialogue boxes, the system can be simulated. The gathering of these components can help to model a large number of architectures.

\section{Results and discussion}

\subsection{Model validation of the excited (S-S) beam}

To validate the results, we compared the results obtained by Dymola/Modelica with those presented in [23] using the finite element method with ANSYS. The various parameters used in this simulation are given in Table 2. The initial conditions for the displacements and their velocities were equal to zero.

The test was performed in order to study the dynamic response of the beam when load was applied. The result of the displacements from Dymola has been presented in 


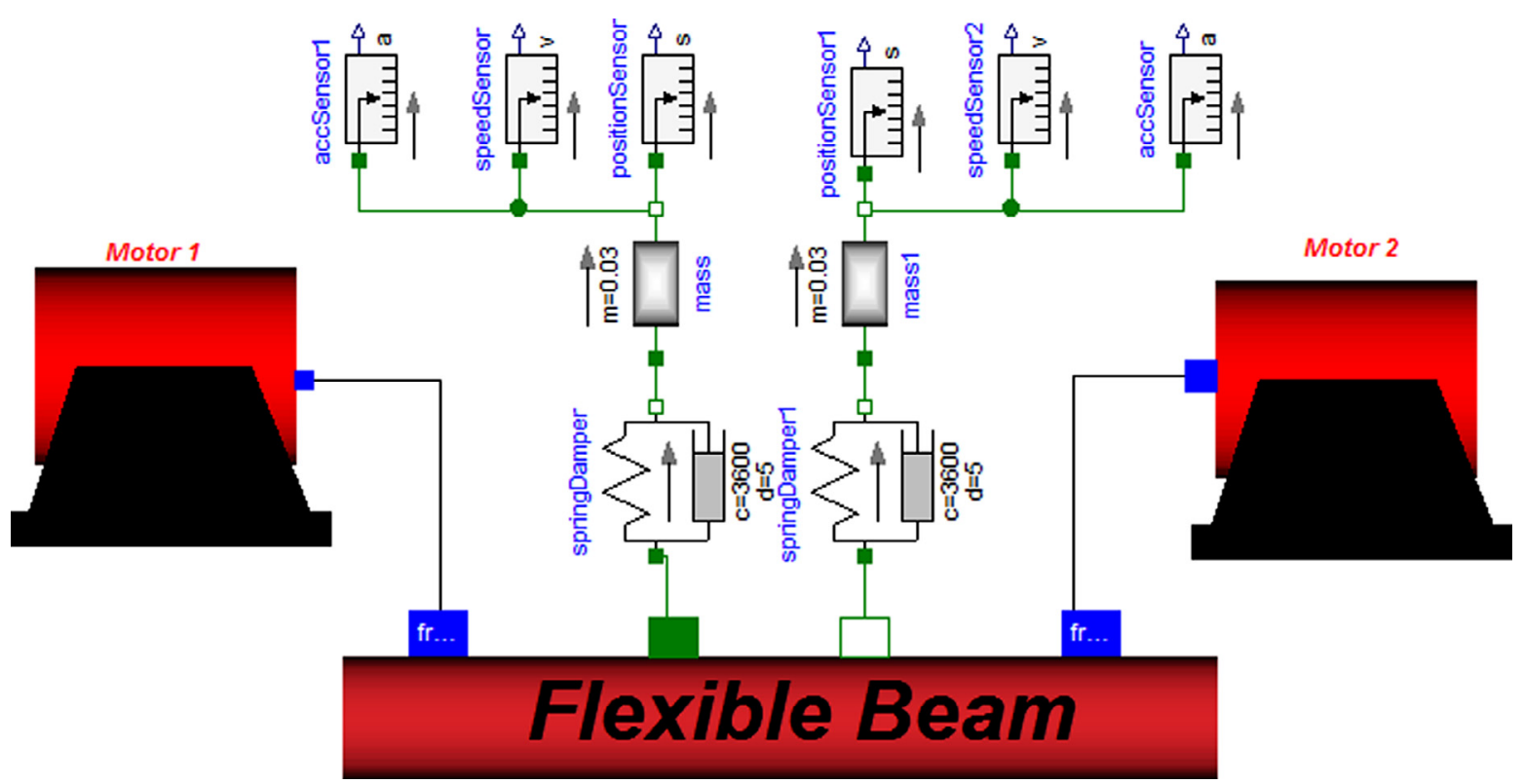

Fig. 4. Model of the system in Dymola/Modelica.

Table 1. New connector properties.

\begin{tabular}{lll}
\hline Type & Name & Description \\
\hline Angular velocity & $z$ & Angular frequency $[\mathrm{rad} / \mathrm{s}]$ \\
Force & $F$ & Force $[\mathrm{N}]$ \\
Position & $y$ & Position $[\mathrm{m}]$ \\
\hline
\end{tabular}

Figure 5 with the same material property and geometry. Comparing the solutions from the two tools (Dymola and ANSYS), we can notice that the amplitudes values were quite similar, enough to be supposed as good result.

\subsection{Model validation of the excited clamped-clamped beam}

The numerical validation of the clamped-clamped (C-C) beam is performed by comparing the results obtained by Dymola/Modelica with those obtained by the model based on the beam component which belongs to the flexible body library of Modelica/Dymola. The beam has the some parameters as those utilized for the validation of the $(\mathrm{S}-\mathrm{S})$ beam.

To validate the analytical model of the $(\mathrm{C}-\mathrm{C})$ beam subjected to a concentrated force in the mid-point, we have developed the setup shown in Figure 6. In fact, the flexible beam element beam is connected at the mid-frame to a concentrated periodic force. The input "sin" has a frequency of $60 \mathrm{~Hz}$ and $10 \mathrm{~N}$ as amplitude. The "Word" component is used to define the gravity direction. In the graphical interface of the beam element, the boundary conditions as well as the geometric and material properties are defined (diameter, length, Young's modulus, Poison ratio, etc.).

Table 3 shows the first five natural frequencies obtained by Dymola (analytic model) and those obtained by Beam based model. It can be seen that the results are very close.
Table 2. Parameters of the mechatronic system.

\begin{tabular}{ll}
\hline Beam length & $l=1 \mathrm{~m}$ \\
Square cross section & $S=0.01 \times 0.01 \mathrm{~m}^{2}$ \\
Young's modulus & $E=2.1 .10^{11} \mathrm{~Pa}$ \\
Material density & $\rho=7800 \mathrm{~kg} \cdot \mathrm{m}^{-3}$ \\
Excitation natural frequency & $f=60 \mathrm{~Hz}$ \\
Force magnitude & $F=10 \mathrm{~N}$ \\
\hline
\end{tabular}

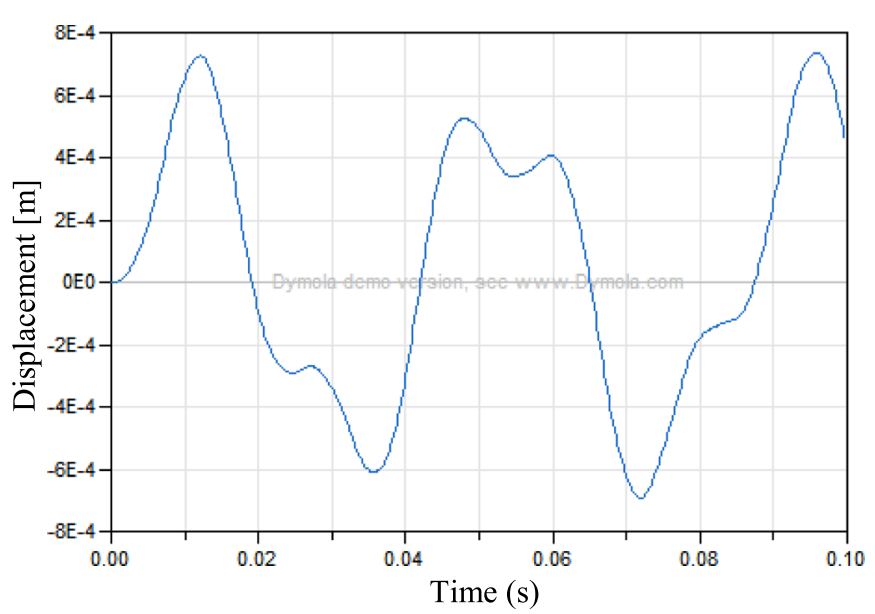

Fig. 5. Displacement in middle point of the beam.

A comparison of the mid-point beam displacement obtained from the two models is shown in Figure 7 . The result shows that the two curves have very close vibration amplitudes and the same oscillation frequency as that of the excitation. Several beating cycles can be seen. The frequency of one complete cycle (beating frequency) is about $7 \mathrm{~Hz}$. The beating phenomenon is related to the 


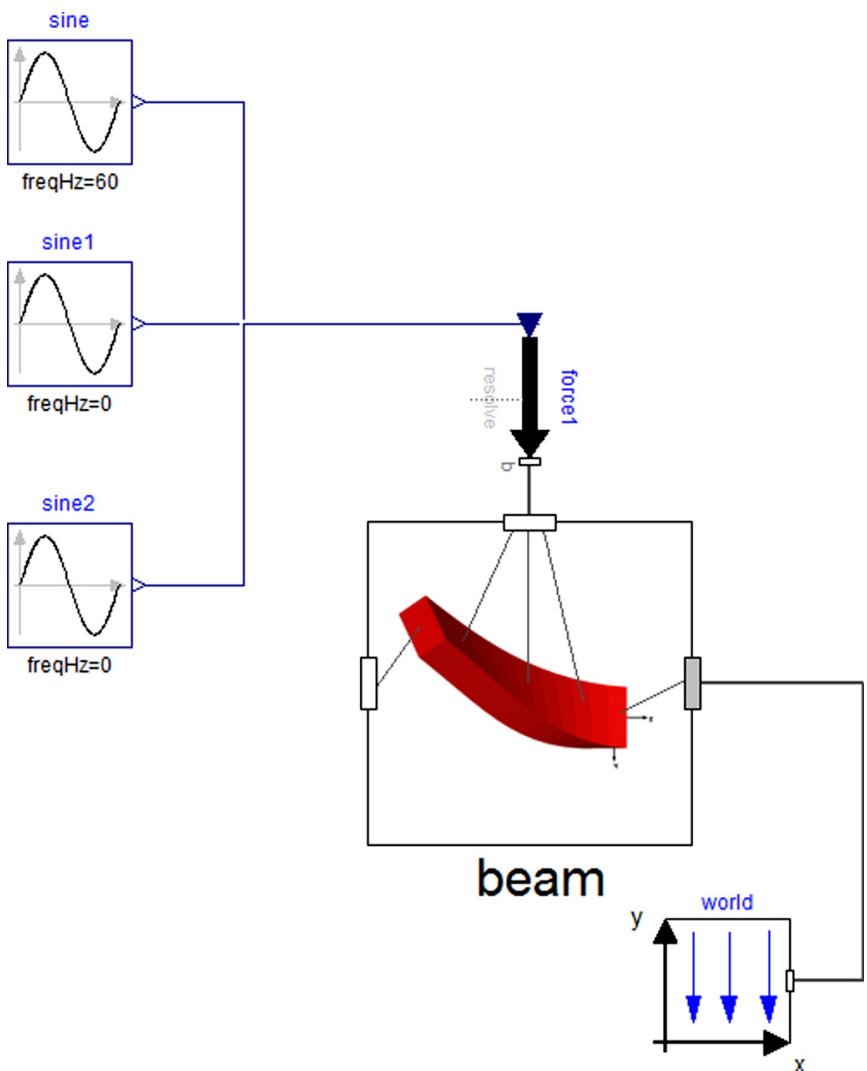

Fig. 6. Configuration of the model validation of the of the (C-C) in Modelica/Dymola.

Table 3. Angular frequency (rad/sec) of the (C-C) beam.

\begin{tabular}{lll}
\hline $\begin{array}{l}\text { Mode } \\
\text { number }(n)\end{array}$ & $\begin{array}{l}\text { Dymola } \\
\text { (flex body) }\end{array}$ & $\begin{array}{l}\text { Dymola } \\
\text { (analytic model) }\end{array}$ \\
\hline 1 & 335,12 & 332 \\
2 & 923.7 & 923.95 \\
3 & 1810.97 & 1810.95 \\
4 & 2993.32 & 2993.62 \\
5 & 4471.95 & 4471.93 \\
\hline
\end{tabular}

closely value the excitation frequency $(60 \mathrm{~Hz})$ with the first natural frequency $(53 \mathrm{~Hz})$. The period of one complete cycle can be computed as follows $T=\frac{1}{60-53}=0.14 \mathrm{~s}$.

\subsection{Parametric study}

In order to simulate the system behavior in terms of parameters and to show how this model can enable designers to visualize the parameters of the system and their dependencies, an analysis has been carried out for different magnitudes.

For simulation purposes, we use the following parameters: the beam has a length $\mathrm{l}=0.5 \mathrm{~m}$ with square cross section $0.01 \times 0.01 \mathrm{~m}^{2}$. The Young's modulus $E=2.1 .10^{11} \mathrm{~Pa}$ and $\rho=7850 \mathrm{~kg} . \mathrm{m}^{-2}$. The beam is subjected to bi-harmonic

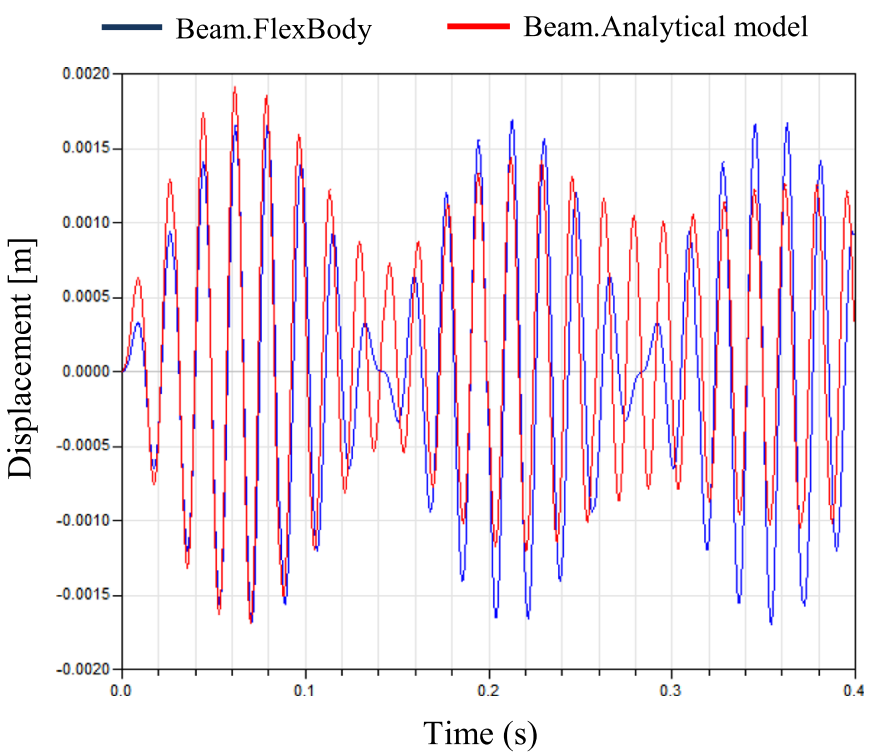

Fig. 7. A plot of mid-point displacements comparison.

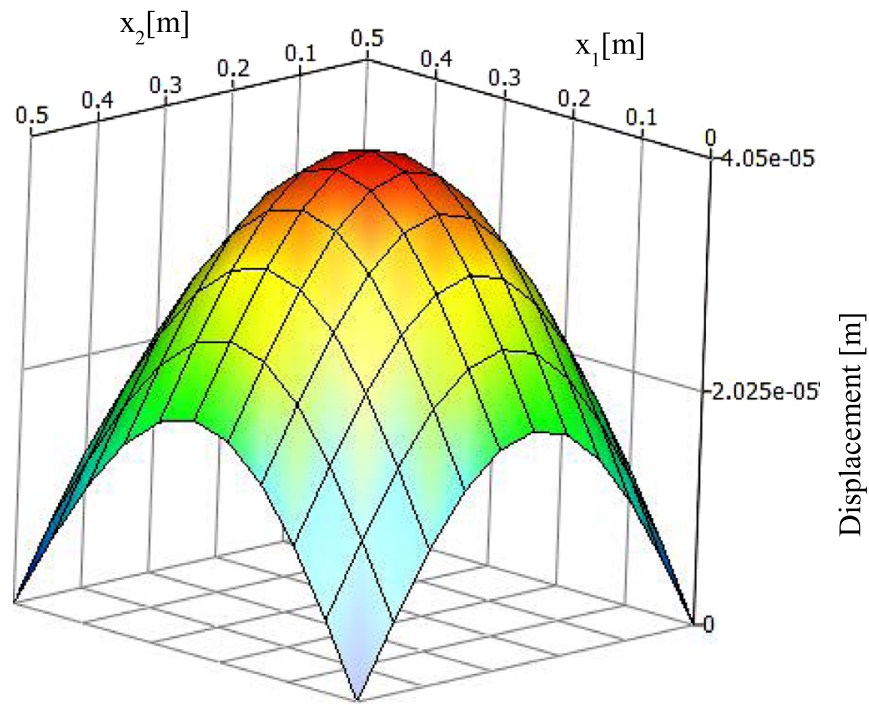

Fig. 8. Displacement at the (S-S) beam center according to the motors positions $\left(x_{1}, x_{2}\right)$.

forces which act at two different positions $x_{1}=0.1 \mathrm{~m}$ and $x_{2}=0.3 \mathrm{~m}$. The excitation amplitudes are $F_{1}=F_{2}=2 \mathrm{~N}$ with excitation frequencies $f_{1}=f_{2}=50 \mathrm{~Hz}$.

According to the theoretical study in Section 2, the electric motors positions on the beam are parametrized. In Figure 8, a two-dimensional plot show the influence of the motors positions on the vibration amplitude at the (S-S) beam mid-point. It can be seen that the more the motors move towards the center of the beam, the grater the vibration amplitude increase.

A comparison of the vibration amplitudes at the (S-S) beam center and the electronic card, measured by the position sensor is shown in Figure 9 given that the card is located at the mid-point of the beam. As it can be observed, the two curves have sinusoidal shapes but they are not in 


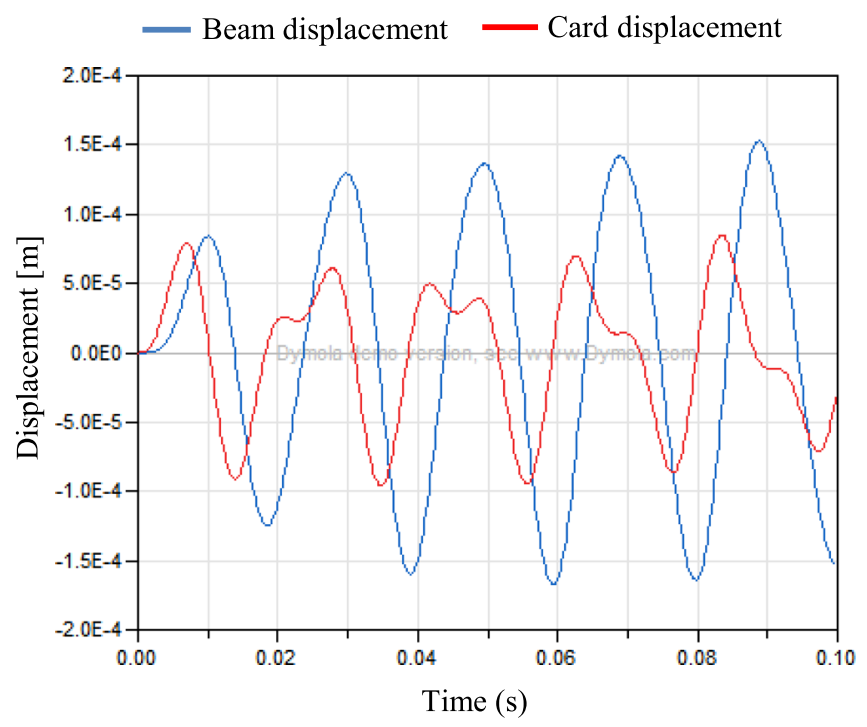

Fig. 9. Displacement at the (S-S) beam center and displacement measured by the position sensor.

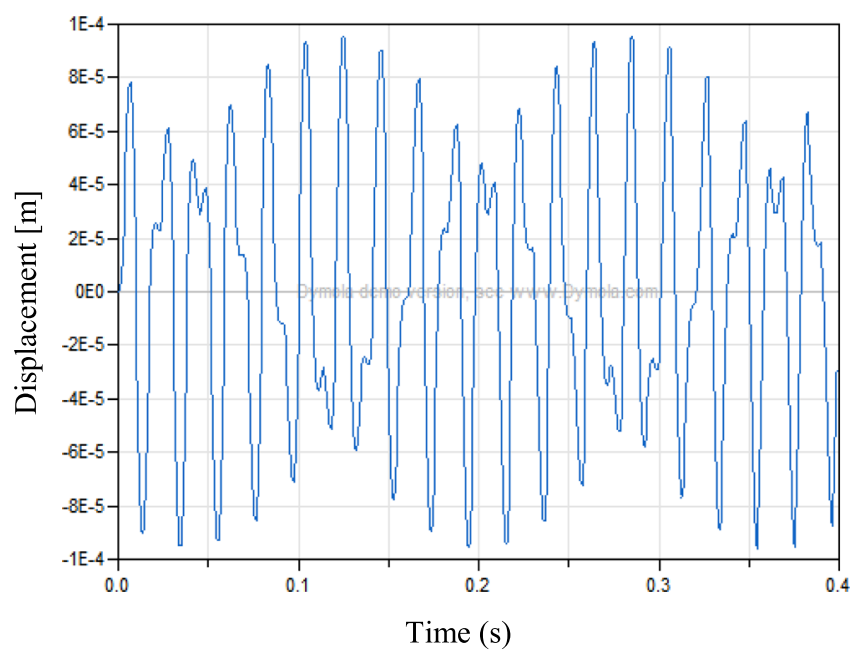

Fig. 10. Displacement of the (S-S) beam mid-point.

phases. This phase shift is due to the card damping effect which introduces a delay of the card displacement sinusoid with respect to the beam displacement sinusoid. On the other hand, the two curves have the same oscillation frequency which is identical to that of the excitations. Using this model, we can measure the movement of the electronic card at any position selected by the user.

The time histories of the measured displacement, speed and acceleration at the center of the (S-S) beam are depicted in Figures 10-12. From these figures, we can found that displacement, speed and acceleration of the beam midpoint have periodic functions shapes with a frequency of $50 \mathrm{~Hz}$ which is the same as that of the excitations frequencies.

Figure 13 depicts the vibrations amplitudes of some points of the beam. In this simulation, one motor is considered $\left(x_{1}=0.25, F_{1}=2 \mathrm{~N}, f_{1}=50 \mathrm{~Hz}\right)$. We find that all these points vibrate in phases with an oscillation frequency

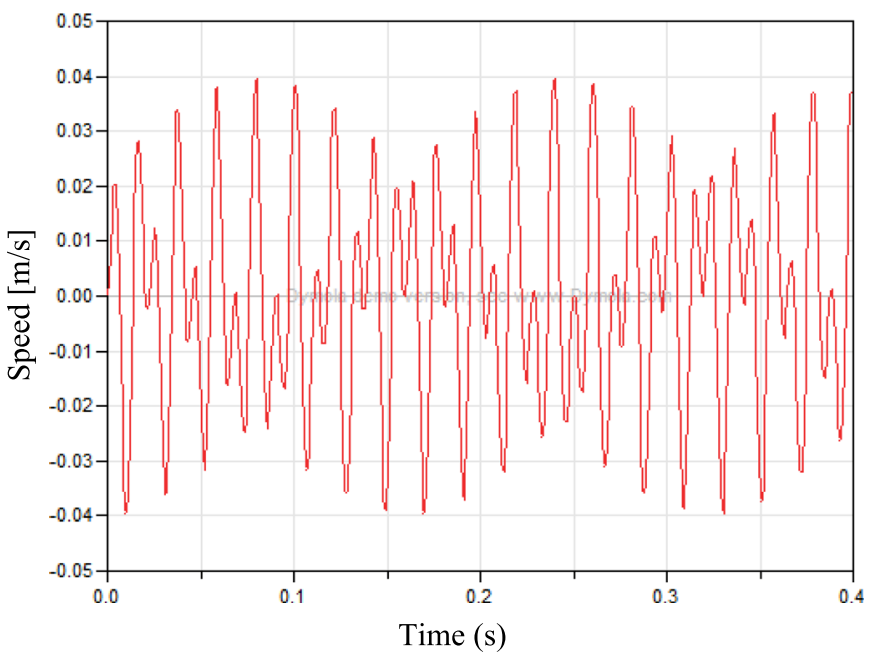

Fig. 11. Speed at the (S-S) beam center.

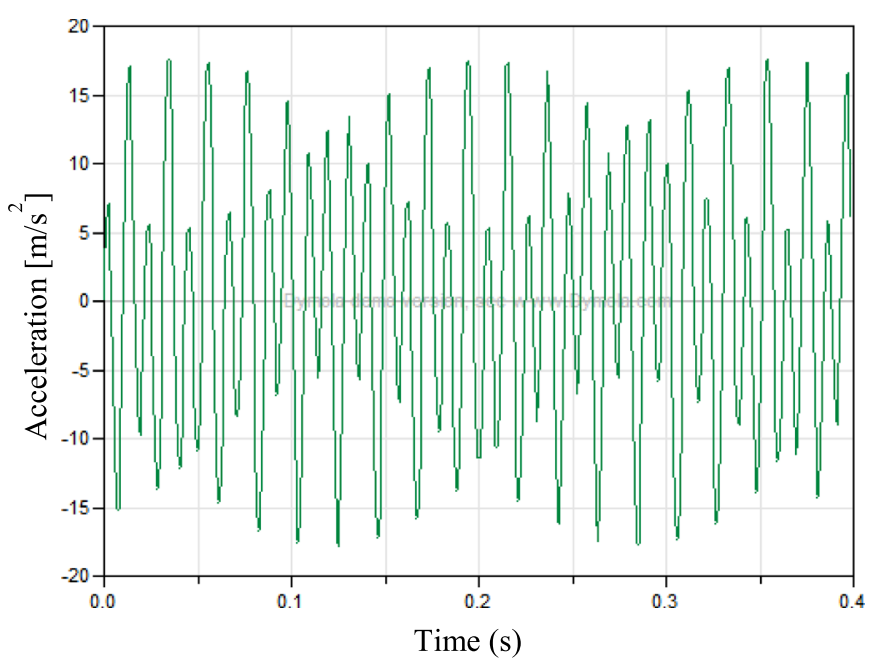

Fig. 12. Acceleration at the $(\mathrm{S}-\mathrm{S})$ beam center.

identical to that of the excitation and the vibration amplitude of the mid-point is the largest.

The influence of the motors excitations amplitudes is shown in Figure 14. This result clearly confirms that the amplitude of vibration increases with the increase of the excitations amplitudes, while keeping the other parameters fixed.

The boundary conditions have a great impact on the dynamic response of the system. Two combinations of boundary conditions are considered in this study, which are (S-S) beam and (C-C) beam. Using this model, designer can study the dynamic response of the system for a chosen type of boundary condition.

Figure 15 shows a comparison of the beam displacement between two types of boundary conditions, which are (S-S) beam and (C-C) beam. As it can be observed, the displacement in the beam center in the case of (S-S) beam is more important than that of the $(\mathrm{C}-\mathrm{C})$ beam. 


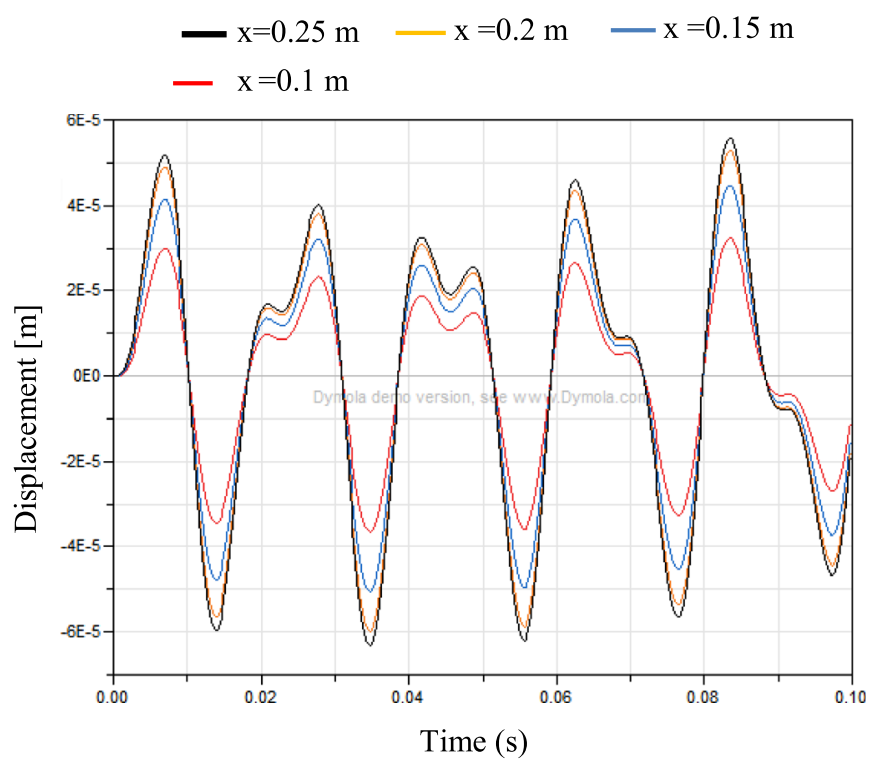

Fig. 13. Displacements of the (S-S) beam (one motor considered).

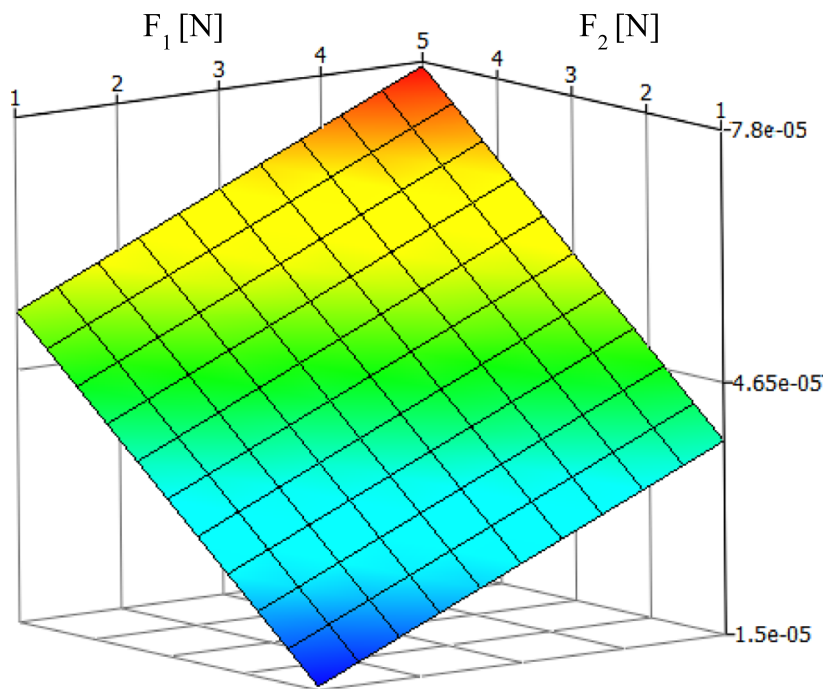

Fig. 14. Displacement at the (S-S) beam center according to the motor excitations amplitudes.

A comparison of the electronic card displacement located on a (C-C) beam with that located on a (S-S) beam is plotted in Figure 16. As it can be seen, the vibration amplitude of the electronic card located on a (S-S) beam is more important than that located on a (C-C) beam. So, by changing the parameters values and the types of boundary conditions, designers can choose between several possible developments and they can also check whether the simulated resulting behavior replicates the desired one.

\section{Conclusions}

In this paper, a new conceptual design methodology to help system architects to define concepts and solutions has been carried out. The object-oriented technology is utilized to create a reusable component-based architecture. In fact,

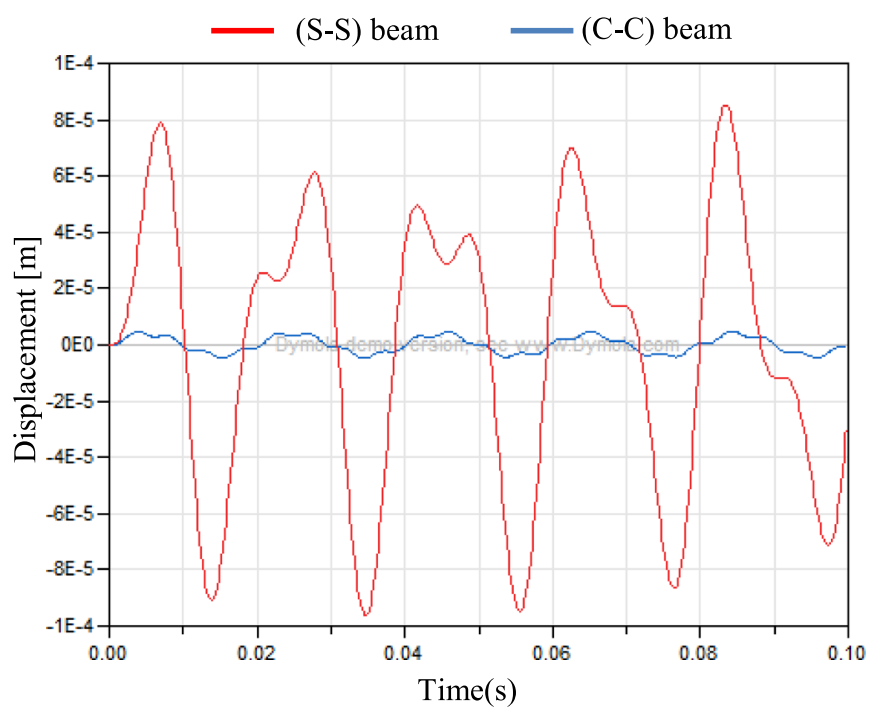

Fig. 15. Displacement at the beam center.

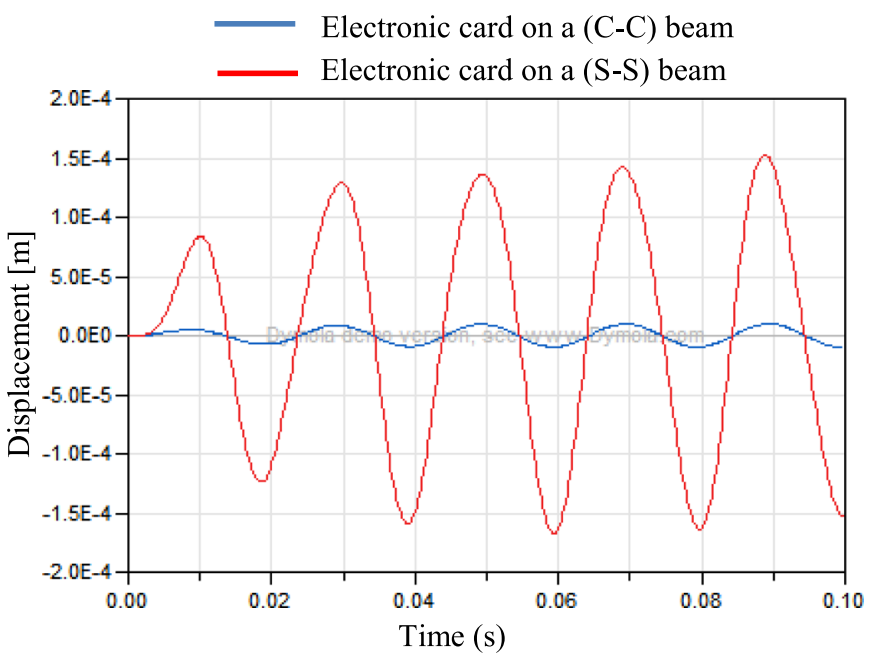

Fig. 16. Electronic card displacement.

the developed model uses standard connector to guaranty the compatibility with Modelica Standard library components. In order to obtain quick resolutions, an analytical approach is adapted using Modelica language. This methodology enables designers to characterize the vibration behaviour of the overall system and to investigate the influence of selected boundary conditions on the dynamic response. This modeling process will help system architects to quickly build up system models, the analysis, and the characterisation of complex multibody systems. A full design may evolve from this work.

\section{References}

[1] R. Barbedienne, O. Penas, J.-Y. Choley, A. Rivière, A. Warniez, F. Della Monica, Introduction of geometrical contraints modeling in SysML for mechatronic design, in: Mecatronics (MECATRONICS), 2014 10th France-Japan/ 8th Europe-Asia Congress on. IEEE, 2014, pp. 145-150 
[2] M. Hammadi, J.Y. Choley, O. Penas, A. Riviere, J. Louati, M. Haddar, A new multi-criteria indicator for mechatronic system performance evaluation in preliminary design level, in: Mechatronics (MECATRONICS), 2012 9th FranceJapan \& 7th Europe-Asia Congress on and Research and Education in Mechatronics (REM), 2012 13th Int'l Workshop on. IEEE, 2012, pp. 409-416

[3] C. Zheng, J. Le Duigou, M. Bricogne, E. Dupont, B. Eynard, Interface model enabling decomposition method for architecture definition of mechatronic systems, Mechatronics 40 (2016) 194-207

[4] L. Wang, W. Shen, H. Xie, J. Neelamkavil, A. Pardasani, Collaborative conceptual design-state of the art and future trends, Comput. - Aided Des. 34 (2012) 981-996

[5] H. Komoto, T. Tomiyama, A framework for computer-aided conceptual design and its application to system architecting of mechatronics products, Comput. - Aided Des. 44 (2012) 931-946

[6] G. Rzevski, On conceptual design of intelligent mechatronic systems, Mechatronics 13 (2003) 1029-1044

[7] R. Scheidl, B. Winkler, Model relations between conceptual and detail design, Mechatronics 20 (2010) 842-849

[8] M. Hammadi, J.-Y. Choley, F. Mhenni, A multi-agent methodology for multi-level modeling of mechatronic systems, Adv. Eng. Inform. 28 (2014) 208-217

[9] T. Habib, H. Komoto, Comparative analysis of design concepts of mechatronics systems with a CAD tool for system architecting, Mechatronics 24 (2014) 788-804

[10] F.A. Salem, Mechatronics design of ball and beam system: education and research, Control Theory Inform. 3 (2013) $2224-5774$

[11] P. Mucci, R. Singh, Active vibration control of beam subjected to AM or FM disturbances, Noise Control Eng. 43 (1995) 159-171

[12] A. Buchacz, Dynamical flexibility of discrete-continuous vibrating mechatronic system, J. Achiev. Mater. Manuf. Eng. 28 (2008) 159-166
[13] A. Buchacz, Characteristics of discrete-continuous flexibly vibrating mechatronic system, J. Achiev. Mater. Manuf. Eng. 28 (2008) 43-46

[14] A. Buchacz, M. Płaczek, Selection of parameters of external electric circuit for control of dynamic flexibility of a mechatronic system, Solid State Phenom. 164 (2010) 323-326

[15] G. Hamza, J.Y. Choley, M. Hammadi, M. Barkallah, J. Louati, A. Riviere, M. Haddar, Pre-designing of a mechatronic system using an analytical approach with dymola, J. Theor. Appl. Mech. 53 (2015) 697-710

[16] G. Hamza, J.Y. Choley, M. Hammadi, M. Barkallah, J. Louati, A. Riviere, M. Haddar, Analytical approach for the integrated preliminary analysis of mechatronic systems subjected to vibration, in: Mecatronics (MECATRONICS), 2014 10th France-Japan/8th Europe-Asia Congress on. IEEE, 2014, pp. 151-155

[17] A.M. Veprik, V.I. Babtisky, Vibration protection of sensitive electronic equipment from harsh harmonic vibration, J. Sound Vib. 238 (2000) 19-30

[18] A.M. Veprik, Vibration protection of critical components of electronic equipment in harsh environmental conditions, J. Sound Vib. 259 (2003) 161-175

[19] S. Mishra, M. Pecht, T. Smith, I. McNee, R. Harris, Remaining life prediction of electronic products using life consumption monitoring approach, in: Proceedings of the European Microelectronics Packaging and Interconnection Symposium, 2002, pp. 136-142

[20] S.S. Rao, Vibration of continuous systems, John Wiley \& Sons, Hoboken, New Jersey, 2007, pp. 317-418

[21] M.I. Sakri, P.V. Mohanram, Experimental investigations on board level electronic packages subjected to sinusoidal vibration loads, Int. J. Curr. Eng. Technol. 2 (2014) 427-431

[22] F. Schiavo, L. Vigano, G. Ferretti, Object-oriented modelling of flexible beams, Multibody Syst. Dyn. 15 (2006) 263-286

[23] M. S. Kozien, Analytical solutions of excited vibrations of a beam with application of distribution, Acta Phys. Pol. 123 (2013) 1029-1033

Cite this article as: G. Hamza, M. Hammadi, M. Barkallah, J.-Y. Choley, A. Riviere, J. Louati, M. Haddar, Conceptual design decision support of a mechatronic system using analytical approach with Modelica, Mechanics \& Industry 19, 103 (2018) 